\title{
INTERVIEW
}

\section{Finding Her Niche: An Interview with Emmanuelle Charpentier}

\author{
Kevin Davies and Emmanuelle Charpentier
}

No matter who writes the story of CRISPR, Emmanuelle Charpentier is one of the undisputed heroines of the saga. The French microbiologist, who just celebrated her 50th birthday, played a critical role in launching the field of CRISPR gene editing while moving between labs and countries in her search for a stable and independent research environment. Her contributions leading up to the classic Science paper in 2012, in collaboration with the Jennifer Doudna lab, have been heralded by the community and celebrated with dozens of prestigious scientific awards.

During a flying visit to New York City (primarily to give a memorial lecture at Columbia University) last autumn, we managed to rendezvous with Charpentier - appropriately in a French bistro-for a candid conversation about her upbringing, research adventures, the CRISPR revolution, and future plans.

(This is a lightly edited version of that conversation; the full interview can be heard at Guidepost.)

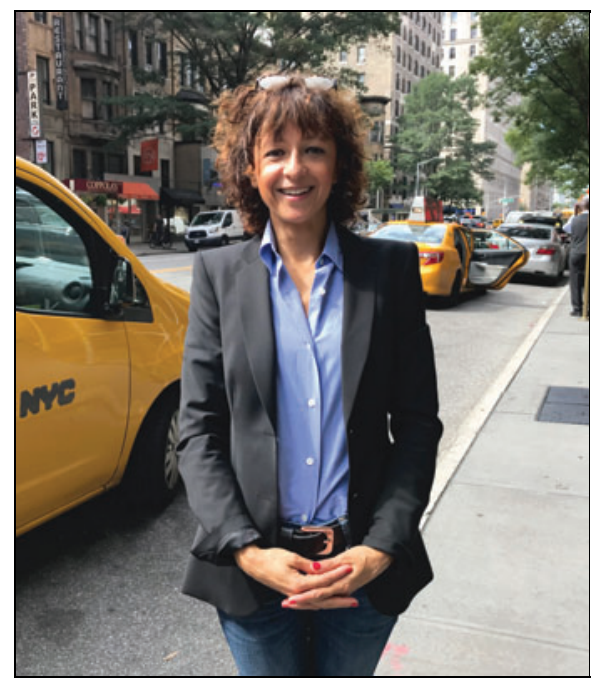

Davies: Was it always your plan to become a microbiologist? I imagine in school you heard about not only the heroes of microbiology but also the great French scientists in bacterial genetics and gene expression. This seems almost like a predestined path?

Charpentier: Yes, I am not sure whether this was my destiny at first. I was always interested early on, starting at six years old, in going to university because of the age difference with my oldest sister. When I was in the second year of primary school, she was going to university. Since that time, I wanted to go to university, too.

I was interested in the global field of sciences, human sciences—sociology, philosophy, psychiatry—and also hardcore sciences, mathematics, biology, and physics. My mother told me when I chose the Pasteur Institute as a host laboratory for my master's degree that when I was 11-12 years old, I came back from school one day and said, "One day I will work at the Pasteur Insti- tute." She reminded me of this when I chose Pasteur for my host laboratory.

Along the way, I became interested more in mathematics, physics, and biology. I chose biology for my specialty for the last year before the baccalaureate. Then I decided to choose life sciences, and then biochemistry, genetics, and microbiology. So, it was a bit of a gut feeling.

The Pasteur Institute is one of the best places in Paris. I was interested in microbes - this was strange because the worst grade I ever had during my undergraduate studies was in a typical microbiology course where you have to learn all the bacterial species. I got my worst grade for this course, and it became my specialty!

Davies: You came to the United States to do a series of postdocs-about six years in the late 1990s. You weren't just working on microbiology, right?

Charpentier: I wanted to focus more on the interactions between bacteria-causing diseases in humans and the

Kevin Davies, Executive Editor, The CRISPR Journal, New Rochelle, New York, E-mail: kdavies@ liebertpub.com; Emmanuelle Charpentier, Max Planck Unit for the Science of Pathogens, Berlin, Germany, E-mail: research-charpentier@mpiib-berlin.mpg.de 
human host. I started a postdoc in the lab of Elaine Tuomanen at The Rockefeller University, working on Streptococcus pneumoniae and diverse aspects of mechanisms of regulation of gene expression that would allow an understanding of how virulence factors and other factors responsible for pathogenicity are produced, specifically in the context of the interaction with human host cells.

When I arrived in New York, they gave me the great news that the lab would be moving to Memphis, Tennessee-to St. Jude-because Elaine was accepting the position of Chair of Infectious Diseases. Even though I went to her lab in Memphis later in 1999 to finish some experiments, I decided in 1997 to start a second postdoc in eukaryotic biology. I understood it was important if I wanted to focus on infectious diseases to give myself the opportunity because I was still young to really understand how to work with eukaryotic organisms.

For this postdoc, I decided to work on skin. During my $\mathrm{PhD}$ thesis, I was trying to understand how antibiotic resistance genes are transferred from clinical isolates to clinical isolates. This type of research allowed me to enter right away into bacterial genetics, the world of plasmids, transposons, phages, horizontal gene transfer. I was focusing mainly on Gram-positive bacterial pathogens causing diseases in humans such as Listeria speciesListeria monocytogenes mainly-but also Streptococci, Staphylococci, and Enterococci.

During my postdoc, I decided to focus on Streptococci because I realized at the Pasteur Institute at the time, research on S. pneumoniae was not represented. There was a group working on Streptococcus pyogenes, but the head of the laboratory was retiring, so I thought it was interesting to focus on those pathogens because, first, they are very fascinating pathogens, but also it would allow me to really find a niche to continue my research in France.

Because I was interested in working on Streptococci and also, to a certain extent, Staphylococci that cause diseases in skin, and I realized that not a lot of knowledge was available for the interactions between these pathogens and skin, I decided to work on skin. Then I said okay, it's best if I spend maybe a year or so in the world of mice because if it is to identify factors on the host side that are responsible for the interactions, it's best to use the proper genetics. At the time, 20 years ago, genetics such as knockouts or transgenics could only be obtained through mouse models.

I figured out the eukaryotic field was in need of better tools; I was working with human pathogens, but there was no possibility of really knocking out genes in human cells. There was just the possibility of overexpressing a gene ectopically in a transient or less transient fashion ... it was the start of trying to improve the protocols to culture human cells, primary cells, skin grafts, etcetera. There was no possibility of doing real genetics.

"There was just the possibility of
overexpressing a gene ectopically in a
transient or less transient fashion ... it
was the start of trying to improve the
protocols to culture human cells, primary
cells, skin grafts, etcetera. There was no
possibility of doing real genetics."

I went back afterwards to the microbiology field and I said, okay, now I need to continue with hardcore research, focus on regulatory mechanisms, and try to find new mechanisms that will be promising in terms of finding new targets for novel anti-infectives and also new ways to target genes and their expression.

This is also the reason why I worked on small RNAs.

Davies: At this point, you've never heard of CRISPR?

Charpentier: In 2002-2003, actually I never heard about CRISPR. From 1999 to 2002, I did a third postdoc focusing on small RNAs and Staphylococci and diverse aspects of cell regulation, replication of plasmids. This was actually the first role found for small RNAs with regulatory functions: an antisense function involved in the regulation of plasmid replication.

I worked on all those aspects, on biofilm formation, on small RNAs in Staphylococci. I worked also on phages. All my time in New York, if I reflect, I strengthened different aspects that really would facilitate my research when I entered the CRISPR field. Along the way, I acquired in my laboratory the techniques that would allow me to really understand the biological questions I was asking with regard to CRISPR and its applications using genetics, biochemistry, and molecular biology.

You know the famous sentence you add at the end of a grant-you hope to find a mechanism that could lead to novel anti-infectives or novel ways to silence genomes and their expression. Thanks to the diversity of the system, I saw right away the potential of a mechanism with CRISPR that would allow novel mechanisms of interactions with proteins and RNA and DNA to be identified, but also I wondered whether this could be a machinery that hopefully would be very attractive for developing gene technologies. 
Davies: When you went from the United States back to Europe, where did you go?

Charpentier: First, I went to the University of Vienna in Austria. This was the start of the Max F. Perutz laboratories. At the time, I could have gone back to Pasteur, although in 2002 at the Pasteur, they were just starting to shape the institute to be able to offer independent positions to marginal leaders. But when I went to Vienna, those positions were not fully shaped yet. For me, it was important to be independent and not to have anyone around me, and just to start my research the way I wanted to start my research.

This is also what experience in the United States brings - those concepts were just starting in Europe. It was important for me to be free. Vienna offered me this possibility, even though I had to struggle quite a bit to get my first funding.

Davies: From Vienna, you moved to Sweden and then subsequently to Germany. This was not some grand plan but something you had to do to keep finding resources to continue your research. Has this has been a handicap?

Charpentier: Yes. In 27 years, I've worked in five countries, seven cities, 10 institutions, 14 different offices, 13 different departments, and 14 apartments. It's a very big turnover!

It was not that it was planned, but I realized during my postdoc that it was going relatively fast. After a short time in a lab, I had seen what I wanted to see, I had done my time, I was successful, I needed to move on. I could feel very quickly the right time to move on, not to get stuck in a position where maybe I would not be able to evolve the way I wanted to evolve. Each time I went somewhere, there was always an incentive or a better position.

Davies: As you look back over the previous 5-10 years, what are one or two of the major insights or turning points that were a tipping point in the story?

Charpentier: There was clearly the experiment-an analysis done in 2009 when we really found out that there was a link between tracrRNA, the second RNA of the CRISPR-Cas9 system, and the CRISPR-Cas9 system. It was a very simple experiment.

The idea I had was that tracrRNA was involved in the activity of the CRISPR-Cas9 system. We had a knockout of tracrRNA because we were studying tracrRNA. (We were going in the wrong direction.) We also had a knockout of CRISPR RNA because we were interested in understanding the CRISPR-Cas9 mechanism. It was a simple experiment when we figured out that when we were knocking out tracrRNA, CRISPR RNA was not maturated any longer, and when we were knocking out CRISPR RNA, tracrRNA was not maturated any longer. We realized right away that Cas9 was important in the stability of those two RNAs.

Very quickly it came to our mind that Cas9 forms a complex with this RNA duplex, finding that indeed tracrRNA can actually form a duplex with CRISPR RNA. I remember it was really the next day, looking at other organisms, figuring out that tracrRNA was actually a unique molecule that was defined not by sequence identity among other tracrRNA molecules in bacteria but a molecule with extremely diverse nucleotide sequence when comparing tracrRNA molecules with one another and with the common characteristic to have an anti-repeat sequence that base-pairs to the cognate repeat of CRISPR RNA.

It was also clear that tracrRNA would not be the end of the story. If you were looking at the other CRISPR-Cas systems-type 1 and type 3 systems, described by the groups of Marraffini with Sontheimer for type 3, also Michael Terns, and for the type 1 system, Stan Brouns and John van der Ost-it was clear that those systems were working with a complex of proteins guided by CRISPR RNA.

The CRISPR-Cas9 system was surely extremely meaningful. In the early work done on the CRISPR-Cas9 system, Cas9 protein was called Csn1, Cas5, Csx12. It was first described in terms of its function by the groups of Horvath and Moineau with Barrangou in the 2007 Science paper. They were working on a strain of Streptococcus thermophilus that actually has the three types of CRISPRCas. It was clear at that time that for the type 2 system, there would be one protein-Cas9-involved for interference. The idea was that CRISPR RNA would be involved and Cas9 would also be involved. Then, Moineau had a breakthrough paper in Nature in 2010 showing that Cas9 can cleave by introducing a double-stranded break on the DNA. This was also very important, but tracrRNA was missing. Moineau's group did the experiments on a genetic level-this was a super-important paper for us (published after we had submitted our first article in Nature that was published in March 2011).

Then, this is what we did, bringing the third component-tracrRNA - into place and showing that, yes, it is an essential component because Cas9 is an enzyme guided by two RNAs.

Davies: It was 2011 when you met Jennifer Doudna?

Charpentier: Yes. The CRISPR field then was fascinating because it was bringing together a very interesting crowd of 
diverse scientists. You had the bacterial geneticists, the molecular biologists, the biochemists, and the structural biologists. There were different papers starting to describe the structures of those proteins, and Jennifer Doudna had already done substantial work on the structure of those proteins.

The structure was really important because we had already identified these diverse Cas9 proteins of different sizes with different sequences, always with a duplex of RNAs but of different structures. My idea was that the structural biology would be important to really understand what is conserved or not in terms of structure on those proteins that have different sizes and sequences. It would be important to gain insight into these mechanisms because if one wanted to reduce the system into practice, then the structural biology might bring clues to shorten the proteins for genetics, especially in higher eukaryotic organisms but also in microbes and do some protein engineering.

When I went to the meeting in Puerto Rico, I knew I would meet Jennifer Doudna. I was looking forward to meeting her because I didn't know her. Also, I presented this work that was published in Nature in 2011. This was at the meeting-the first official CRISPR meeting that was organized, in Wageningen, the Netherlands, by John van der Oost. I figured out that the CRISPR scientists knew one another because they were invited to this secretive meeting that took place in Berkeley prior to 2010 where I was not invited because no one had any idea that I was working on CRISPR.

When I presented my work in October 2010, Jennifer was not present at the meeting. But they discovered that I was working on CRISPR, and they discovered the famous story of tracrRNA. It was very nice because this was, I think, the highlight of everything. The pioneers all came up to me and shook my hand and said, "I think you got the story!"

"But they discovered that I was working on CRISPR, and they discovered the famous story of tracrRNA. It was very nice because this was, I think, the highlight of everything. The pioneers all came up to me and shook my hand and said, 'I think you got the story!'"

So yes, indeed, I had in mind to approach Jennifer and to ask her whether she would be interested in deciphering the structure of Cas9.
Davies: Your collaboration clicked straightaway?

Charpentier: Well, yeah, you meet and sometimes you feel that it is going to work out, sometimes you think it is not going to work out. What was very important for this collaboration is that you can have a good gut feeling and a good interaction with a colleague. But for the lab scientists who are going to work on the project, there is a need to get along with one another. This was very interesting with my students, Krzysztof Chylinski and Martin Jinek, because they were getting along with one another. Krzysztof is polish, and Martin is from the Czech Republic. They could understand one another a little bit when they were speaking Polish and Czech.

We held a number of Skype meetings; I was joining Krzysztof and Martin most of the time. The biological questions were very straightforward. What was important first was to have the Cas9 purified, and then I had all the planning in my lab for the series of assays that we wanted to run, which is, plus/minus tracrRNA, CRISPR RNA, showing that it is cleaving, looking at whether it has any other activity than cleaving, looking for the PAM requirements, looking for the sequence and domain requirements, reducing to practice. And this became the Science paper. Along the way, Martin was trying to improve the conditions to get the CRISPR structure, which ended up in a publication.

Davies: Was there a eureka moment along the way?

Charpentier: For me, no, but there was a second eureka moment after this Northern blot analysis that indicated that really there is an interaction, etcetera. A follow-up observation within the next day after the Northern blot experiment told us everything, which is also a study that ultimately we continued in collaboration with the group of Jörg Vogel in Germany.

A second eureka moment in my lab was when my student Krzysztof showed that indeed, Cas9 is cleaving with tracrRNA and CRISPR RNA. This was it. In the test tube, we have those three components, and it is cleaving. The first assay that we did is actually the first figure of the Science paper, so all the controls were working right away.

The fact that CRISPR would surely be useful as a gene technology, this came earlier, at least for me-even the idea that it could be useful for the silencing and recombination of genomes in higher organisms that are for the treatment of human genetic disorders through new ways to develop gene therapies.

But this was also because prior to starting my master's studies in microbiology at the Pasteur Institute, I had 
done a Bachelor thesis in a lab focusing on human gene therapies in the early 1990s. This was one of my early interests. That is why I started with bacterial genetics, and genetics has always been the "red thread." I developed genetic tools to have better tools to do bacterial genetics when I was a postdoc in the United States. So, it has always been an obsession.

Ultimately, we developed better tools for S. pyogenes, and these tools were very useful to make sure that we understood the right role of tracrRNA and CRISPR, etcetera. These were really tools that were allowing us to do precise genetics.

Davies: In your lecture at Columbia you talked about luck and serendipity in the choice of organism that you worked on.

Charpentier: Serendipity ... we are really interested in my lab in working with $S$. pyogenes because it is a relevant clinical pathogen causing a wide range of diseases. The advantage of $S$. pyogenes Cas9 is that it is working with a very small PAM, Ngg, which gives more flexibility to use this Cas9 protein in a wider way because it offers more possibilities to target DNA sequences. Also, it ended up being the protein that showed the most efficiency in human cells.

We could have worked on another ortholog and maybe this ortholog would have been tested. Scientists would have said, "Yes, okay, but not great." Maybe I would not be here talking to you!

Davies: Much was made upon the publication of the Science paper-particularly in retrospect-that the big question remaining was to demonstrate that this gene editing system would work in human cells. Did you feel that was a formality?

Charpentier: For me, it was not a concern because of other systems that have been used to do knockout mice with proteins originating from bacteria. It was working so well in vitro and then you test it in bacteria, in higher organisms. So, I was very positive [laughs]. Maybe it is my way of approaching science.

Davies: Did you quickly see the potential of this technology and decide to launch a biotech company? Or was it a case of people coming to you?

Charpentier: No. This was 2012 and very much thought through because of my early idea. When I was in the United States (at the end of the 1990s), I got very inter- ested in principal investigators who were trying to look for ways where they could be involved in biotech companies. The vector system I developed when I was in the Novick lab at the Skirball Institute, NYU, was ultimately commercialized. I always had in mind that one day it would be nice if my research could lead to antiinfective strategies. Indeed, there is a way to harness CRISPR-Cas9 in this regard, at least as a sort of antibacterial. But the right application for me was human gene therapy.

\section{"I always had in mind that one day it} would be nice if my research could lead to anti-infective strategies. Indeed, there is a way to harness CRISPR-Cas9 in this regard, at least as a sort of antibacterial. But the right application for me was human gene therapy."

In 2012, my lab was also right in the middle of a new move and so very busy. I contacted Rodger Novak, who put me in contact with an investor he knew, Shaun Foy. I just told him, "I'm working on CRISPR." I explained to him that there is a possibility of applying the technology directly in human gene therapy. "Maybe you think I am crazy. I do not know timings, etc." This was the start of CRISPR Therapeutics and ERS Genomics, with first a wish to establish the company in the United Kingdom or in Switzerland.

In early 2013, I approached Jennifer Doudna and told her that I was more interested in going toward therapeutics. She was more interested in the toolbox, kits, and so on. I also approached Feng Zhang at that time who said he would be interested in joining the company. But ultimately, the Broad went in another direction. We followed our own way.

Davies: We are on the verge of entering the clinic. The literature is full of concerns, scares, off-target effects, p53, and immune effects. Do you feel that delivery may be the biggest challenge ahead of meaningful therapeutic benefit using CRISPR gene editing?

Charpentier: Yes, I think so. As much as it is important to work on the specificity of CRISPR-Cas9, the bottleneck remains the delivery systems to really have CRISPR-Cas9 delivered in the maximum number of 
cells in the most efficient way, in the safest way, with no secondary effects or unwanted reactions.

Davies: What is the main focus of your group in Berlin now?

Charpentier: This is really understanding mechanisms of regulation of gene expression, mainly in S. pyogenes in the context of the human host and in the context of all stresses that $S$. pyogenes may encounter. We have got some different aspects of gene regulation at the transcriptional/post-transcriptional, translational/post-translational level. We are interested in the class of small regulatory molecules, RNAs, proteins interacting with RNAs, degradation and maturation of RNAs, small RNAs, and trying to find their targets.

We are also interested in all the pathways of toxin/ antitoxin systems. We are interested in protein quality control and proteins named Clps that are chaperone proteins with unfoldase functions. We are also interested in CRISPR, surely - the biochemistry of CRISPR-Cas9, but also the biology of other CRISPR-Cas systems.

Another line of research that will start soon is a CRISPR-Cas application on the development of infectious models. We are also interested in host-pathogen interactions with innate immunity to a certain extentspecifically how the host reacts to $S$. pyogenes that produces small bacterial visitors, extracellular structures produced by $S$. pyogenes triggering functions with regard to infections.
Davies: You've been in Berlin now for three years. Are you happy there?

Charpentier: Yes. I joined the Max Planck Institute for Infection Biology three years ago, and now I have started a new small institute focused on the science of pathogens. We've been in negotiation to find space in Berlin near the campus of Humboldt University and the University Hospital La Charité, so we hope to start planning the building soon. We hope that the building will be ready in five to six years, where we'll have the opportunity to recruit five or six independent group leaders to focus the research of the institute on the science of pathogens.

Davies: Your life has changed almost beyond recognition, I suspect, in the past five years. Do you wish sometimes you could go back to the relative anonymity-to just be in the lab and put the radio on and do your work?

Charpentier: No, I don't have that luxury anymore. I am conflicted. Let me put it this way: I have so many things to take care of that, in a way, I have to remain pragmatic. I try to organize myself as much as I can to deal with the overwhelming situation on different angles, whether it is the CRISPR-Cas side effects or the challenge that I have encountered lately - establishing an independent institute in an independent locality. This was the reason I started this new institute.

But sure, I miss the time where I could be in the lab and a little bit freer, quieter, being able to focus on research. 